\title{
SENSOR WEB TECHNOLOGY CHALLENGES AND ADVANCEMENTS FOR THE EARTH SCIENCE DECADAL SURVEY ERA
}

\author{
Charles D. Norton ${ }^{1,3}$ and Karen Moe $e^{2,3}$ \\ ${ }^{1}$ Jet Propulsion Laboratory \\ California Institute of Technology, Pasadena, California USA \\ Charles.D.Norton@jpl.nasa.gov \\ ${ }^{2}$ National Aeronautics and Space Administration \\ Goddard Space Flight Center, Greenbelt, Maryland USA \\ Karen.Moe@nasa.gov \\ ${ }^{3}$ National Aeronautics and Space Administration \\ Earth Science Technology Office, USA
}

\begin{abstract}
This paper examines the Earth science decadal survey era and the role ESTO developed sensor web technologies can contribute to the scientific observations. This includes hardware and software technology advances for in-situ and in-space measurements. Also discussed are emerging areas of importance such as the potential of small satellites for sensor web based observations as well as advances in data fusion critical to the science and societal benefits of future missions, and the challenges ahead.
\end{abstract}

Index Terms- Sensor web

\section{INTRODUCTION}

The U.S. National Research Council Earth Science Decadal Survey recommends that NASA pursue a set of 15 on-orbit missions, implemented across a 3 tiered timeline, to measure fundamental Earth system science parameters essential to monitoring and data record enhancement of the forcings and responses related to climate change. Sensor web systems can provide a means to address the requirements of the Decadal Survey including rapid response and decision support, as well as understanding the implications of complex phenomena interactions via integrated observations from multiple vantage points. Significant progress has been made in the development and deployment of sensor webs for a variety of application areas [1]. In this paper we will identify and explore the key technological challenges and advancements needed to increase the adoption of sensor webs to the current needs of the Earth science decadal survey missions and related systems during this era. These are high data-rate measurement missions of multiple geophysical parameters. The Decadal Survey identifies several societal benefit areas where there is a need for timely acquisition of remote sensing observations and delivery of data products to support public health and safety needs. Sensor Webs, for example, have successfully demonstrated the capability to support the societal benefits in response to natural hazards [2].

In the following sections we discuss several examples of sensor web applications for decadal survey missions, drawn primarily from technology investments from the NASA Earth Science Technology Office.

\section{SENSOR WEB CHALLENGES DRIVEN BY THE DECADAL SURVEY}

NASA's Earth Science Technology Office (ESTO) has been investing in the development of sensor web technologies to support the Decadal Survey and other scientific objectives. One of the challenges identified is the need to support insitu ground-based calibration/validation campaigns for the spaceborne missions, for example SMAP - a soil moisture radar/radiometer measurement mission [3]. While there are other active and passive remote sensing missions that can benefit from ground-based sensor webs for calibration, as well as advances in wireless sensor networks to enable distributed data collection and distribution, one challenge that has yet to be investigated in depth is how to perform data fusion among the spaceborne and ground-based assets [4]. This will be a key criterion to ensuring that sensor webs are viable as a calibration source for spaceborne instruments as well as the role they can have to contribute to improving the quality and reducing the uncertainties in decadal survey observations and related science. A related challenge in the CLARREO mission, which will produce highly accurate measures of solar reflectance, is the need to coordinate 
among flight missions to acquire coincident measurements for inter-spacecraft calibration. Again, sensor webs provide mechanisms and tools to simplify the data acquisition process [5].

ESTO has also developed technology in another critical usage area for sensor webs - infrastructure for sensor web systems to support model interactions, specifically for mission design and science observation planning i.e. for radar surface deformation measurements such as the DESDynI mission [6]. Related technology development for airborne platforms and UAVs is on going and one can conceive of multiple UAVs utilizing such technology in a sensor web to contribute to regional observations [7].

Looking even further ahead small satellites, such as CubeSats, are attractive low-cost platforms to get small payloads to orbit quickly and in large numbers. Advancements in compact instrument technologies, communications, and availability of launches make these an ideal platform as a spaceborne sensor web for scientific observations and technology validation. While ESTO is currently investing in CubeSat technology validation projects for the ACE [8] and HyspIRI decadal survey missions, in the future one can foresee how these platforms can serve as precursors to help establish and refine observation requirements for future missions. Indeed they can also be the basis for small satellite based Earth observation sensor webs/constellations themselves to complement the decadal survey measurements.

The following subsections explore sensor web challenges in these areas, with subsequent advances, in more depth.

\subsection{Sensor Web Challenges for Active Measurements}

The QuakeSim project has developed a solid Earth science framework to model, characterize, and understand earthquake and active tectonic processes [9]. This necessitates integration of many observational measurements and models to quantify the earthquake process. These processes occur over time and physical length scales that are both long and short so a host of in-situ, airborne, and spaceborne observations are essential to acquiring a range of data to study this complex interacting system and to produce hazard forecasts of scientific and societal benefit.

Fig. 1 shows a sample of the kind of product QuakeSim can produce while hinting at how a sensor web can further enhance data acquisition and understanding of events such as the M7.2 Baja-Mexico El Mayor-Cucapah earthquake of April 4, 2010. As described, data from multiple sources can be integrated into an earthquake simulation and forecasting system. With the addition of UAVSAR measurements radar
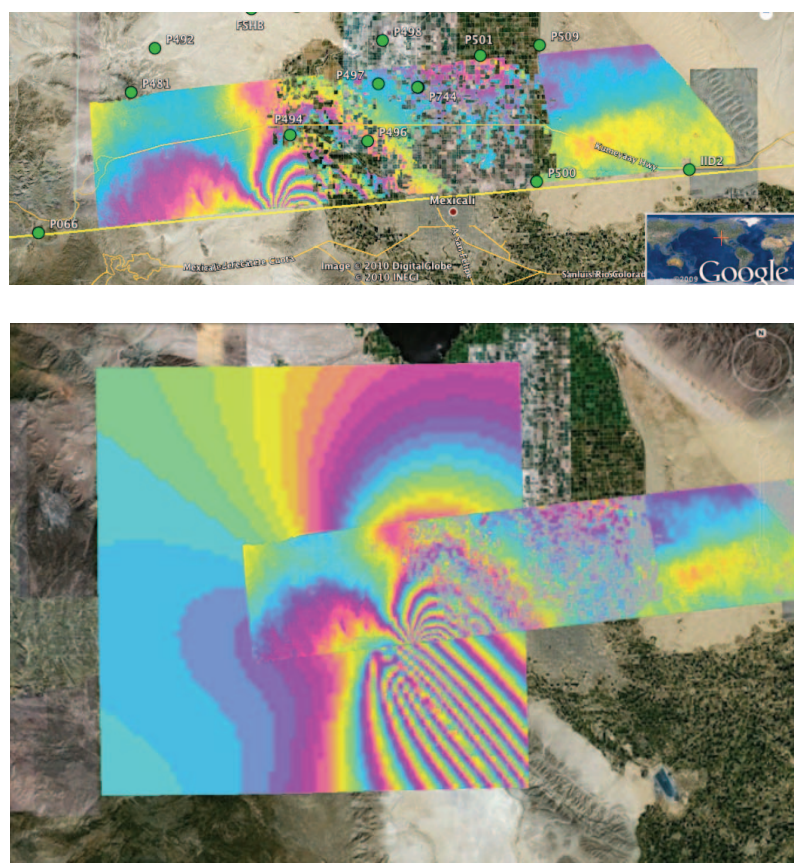

Figure 1. El Mayor-Cucapah earthquake deformation. Top: UAVSAR coseismic image of phase due to coseismic deformation, with (green dot) locations of Plate Boundary Observatory GPS stations (Courtesy UNAVCO). One color cycle corresponds to $12 \mathrm{~cm}$ of relative ground motion toward the UAVSAR instrument. Bottom: Display of synthetic interferogram ground motion inversions (from Disloc) overlaid by observed UAVSAR interferogram on Google Earth [9]. This illustrates the benefit of integrating data from airborne and in-situ sensors with model results to characterize fault behavior and deformation effects where long-term data records produced by a sensor web of repeated observations from multiple vantage points could improve geophysical understanding.

interferegrams are now integrated within QuakeSim modeling applications.

To capture the physics and dynamics to improve forecast models global and continuous observations are needed from space, as well as regional measurements from ground and airborne sensors with a cadence that allows for near real-time assimilation of the combined observations into systems such as QuakeSim. This is particularly important to detect precursors of large events and to improve models based on capturing geophysical dynamics immediately after large events occur. While deployment of the proper number and variety of assets is an obvious challenge such a sensor web can only be effective if the data system infrastructure is developed and deployed to rapidly collect, analyze, and potentially redirect assets to resolve dynamically changing phenomena of interest with a community-defined way of sharing the results - a challenge the Quakesim team is currently undertaking. 

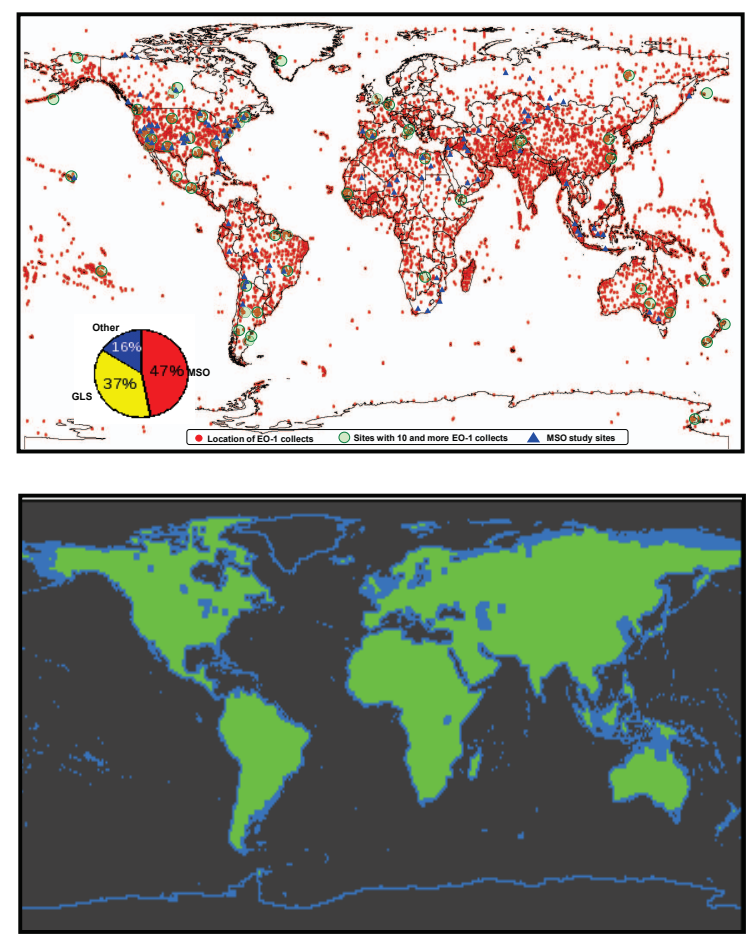

Figure 2. Hyperion/HyspIRI Global Coverage vs. Time. For climate contributions global coverage is required with a revisit of less than 20 days. Top: The EO-1/Hyperion sampling mission coverage locations over 10 years. Bottom: Complete terrestrial coverage every 19 days by the proposed HyspIRI mission [10]. This demonstrates the sensor web "like" potential of frequent observations where the Intelligent Payload Module (IPM) allows for near real-time quick-look product generation and transmission among distributed direct broadcast ground stations.

\subsection{Sensor Web Challenges for Passive Measurements}

HyspIRI is a proposed global mapping mission measuring land and shallow aquatic habitats at $60 \mathrm{~m}$ and deep oceans at $1 \mathrm{~km}$ resolution every 5 days with a thermal IR imager (TIR) and every 19 days with a visible to near-IR spectrometer (VSWIR). While not a sensor web mission, HyspIRI exhibits related characteristics (and identifies related challenges) as these two primary science instruments will provide unprecedented coverage compared to past imaging spectroscopy missions as exhibited in fig. 2 .

One of these challenges is data volume; HyspIRI will acquire approximately $4.5 \mathrm{~TB}$ of data per day and roughly 5 $\mathrm{PB}$ of data over the nominal mission lifetime. As a global mapping mission HyspIRI has a requirement to transfer data to a science processing center within 2 weeks of downlink. There is a capability, however, to satisfy high-priority requests for a limited volume of regional data within 6-48 hours of downlink via the Intelligent Payload Module (IPM) for a real-time subset/product solution. The IPM, given the
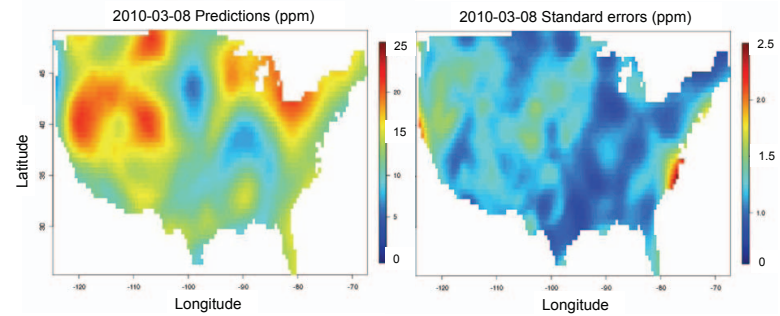

Figure 3. Space/Time Statistical Inference Data Fusion. Results of space/time data fusion to estimate lower $\mathrm{CO} 2$ for one time period. Left: $\mathrm{CO} 2$ estimates. Right: Associated uncertainties. This illustrates one of the future challenges sensor webs will need to resolve to integrate measurements simultaneously from multiple sources to produce scientific results of interest [11].

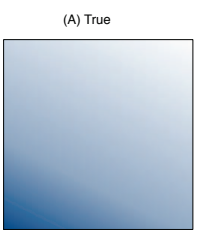

(A) True

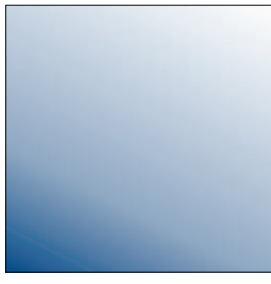

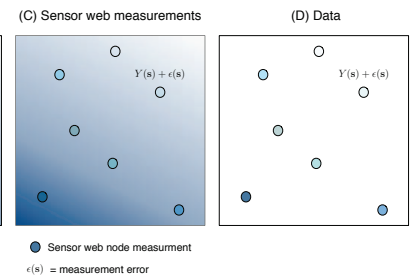

O Sensor web node location
$Y(s)=$ true value at location $s$

(D) Data



Figure 4. Challenges in Sensor Web Data Fusion. The challenge is to infer the true field (A) from the data (D) that is sparse and exhibits instrument error and biases [11].

repeat frequency of HyspIRI, will require capabilities and face challenges similar to what a sensor web might see. The ability to rapidly and autonomously detect and respond to changes in events, communicated over a latency and bandwidth limited network, will be required where frequent global observations are taken. Indeed, it would take EO1/Hyperion (albeit a sampling mission) 100 years to perform the observations HyspIRI will provide in 1 year. For such reasons, a high data rate direct broadcast capability is one challenge a sensor web for global continuous measurements needs to overcome.

\subsection{Data Fusion for Sensor Web Observations}

For sensor webs to have an impact, mechanisms must be introduced to fuse data from multiple sources at varying resolutions in time. This must be done in a systematic way 


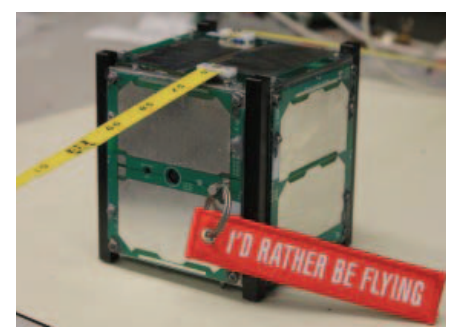

Figure 5. The M-Cubed/COVE $1 U$ CubeSat. This project will validate technologies for the ACE decadal survey mission [8], but similar efforts are also laying the groundwork for future lowcost and rapidly deployable sensor web technologies. Industry and academia are planning future missions using multiple CubeSats for collaborative observations. Challenges in downlink bandwidth, data fusion, and constellation control must be solved as system capabilities rapidly expand over the coming years.

where uncertainties in estimates of physical parameters are clearly captured and understood in the context of sparse or biased observations. Even with near continuous global coverage from a sensor web there will be locations where observations are missed, or not trusted, and there will also be bias errors from "identical" instruments flying or placed in-situ on different platforms.

ESTO is investing in data fusion methodology techniques based on statistical inference, e.g. fig. 3, to advance the ability to produce better estimates of physical phenomena from instrument observations with uncertainties [11]. While also important for missions with a single instrument performing spatial/temporal sampling this kind of technology is addressing the challenges that must be solved to make sense of data from multiple sensor web observations as well, e.g. fig. 4.

\subsection{Small Satellites for Sensor Web Observations}

Looking ahead, small satellites have demonstrated that science and technology validation can be performed using inexpensive, fast-turnaround, and highly deployable systems where the impacts of occasional failures can be tolerated and mitigated quickly. For instance QB50, a proposed international network of 50 CubeSats to measure the thermosphere, is on going. The future of sensor webs will be based on these systems, and challenges in data systems and communications will need to be mitigated as the community scales up from current designs, e.g. fig. 5, to such platforms.

\section{CONCLUSIONS}

An important goal of the Decadal Survey is to establish a series of measurements that can be fused to characterize interactions, forcings, and responses among multiple key parameters that drive the Earth system. Technologies supporting these goals are also helping to solve challenges of future sensor web systems that in turn will form the next set of future missions supporting continuous and global observations in Earth science.

\section{ACKNOWLEDGEMENTS}

We deeply acknowledge the work of the AIST principal investigators that are pursuing these advancements on behalf of NASA ESTO.

\section{REFERENCES}

[1] L. Di, K. Moe, and T. L. van Zyl, "Earth Observation Sensor Web: An Overview," IEEE Journal of Selected Topics in Applied Earth Observations and Remote Sensing (JSTARS), Vol. 3, No. 4, IEEE, pp. 415-417, December 2010.

[2] D. Mandl, et al., Sensor webs with a Service-Oriented Architecture for On-demand Science Products, SPIE Conference, San Diego, CA, August 2007 [6684-40].

[3] M. Moghaddam, et. al, "A Wireless Soil Moisture Smart Sensor Web Using Physics-Based Optimal Control: Concept and Initial Demonstrations," IEEE Journal of Selected Topics in Applied Earth Observations and Remote Sensing (JSTARS), Vol. 3, No. 4, IEEE, pp. 522-535, Dec. 2010.

[4] E. L. Kang, N. Cressie, and T. Shi, "Using Temporal Variability to Improve Spatial Mapping with Application to Satellite Data", Canadian Journal of Statistics, 2010.

[5] M. Lee, R. Weidner, K. Bowman, A. Sandu, K. Singh, "Sensor-web Operations Explorer" at 2008 AGU Fall meeting, Dec, 18, San Francisco, CA.

[6] A. Donnellan, J. Parker, C. Norton, G. Lyzenga, M. Glasscoe, G. Fox, M. Pierce, J. Rundle, D. McLeod, L. Grant, W. Brooks, T. Tullis, "QuakeSim: Enabling Model Interactions in Solid Earth Science Sensor Webs," IEEE Proc. 2006 Aerospace Conference, DOJ 10.1109/AERO.2007.353087.

[7] Y. Lou, S. Chien, D. Clark, J. Doubleday, R. Muellerschoen, S. Saatchi, D. Tran, and Y. Zheng, "A Forest Fire Sensor Web Concept with UAVSAR," IGARRS, Boston, MA, 2008.

[8] P. Pingree, T. Werne, D. Bekker, T. Wilson, J. Cutler, M. Heywood, "The Prototype Development Phase of the CubeSat Onboard processing Validation Experiment (COVE)," IEEE Proc. 2011 Aerospace Conference, Big Sky, MT (to appear).

[9] A. Donnellan, et al., "QuakeSim: Increasing Accessibility and Utility of Spaceborne and Ground Based Earthquake Fault Data," NASA Annual Report, 2011 Report from the ESTO Advanced Information Systems Technology (AIST) Program. January 2011.

[10] Decadal Survey Tier 2 Mission Study Summative Progress Report for HyspIRI, NASA HQ, Washington, D.C., Nov. 2010.

[11] A. Braverman, "Geostatistical Data Fusion for Remote Sensing Applications", NASA Annual Report, 2011 Report from the ESTO Advanced Information Systems Technology (AIST) Program. April 2011. 